\title{
Systematic review on the needle and suture types for uterine compression sutures: a literature review
}

Shinya Matsuzaki ${ }^{1 *}$ D, Mariko Jitsumori ${ }^{1}$, Takeya Hara', Satoko Matsuzaki², Satoshi Nakagawa', Tatsuya Miyake', Tsuyoshi Takiuchi ${ }^{1}$, Aiko Kakigano ${ }^{* *}$, Eiji Kobayashi', Takuji Tomimatsu ${ }^{1}$ and Tadashi Kimura ${ }^{1}$

\begin{abstract}
Background: This study aimed to identify and review associations between the types of sutures used for uterine compression suture (UCS) and its outcomes in postpartum hemorrhage.

Methods: An electronic search using PubMed and Scopus databases was performed. We included the English articles reported from January 1, 1997, to May 31, 2017, using search words or terms regarding the types of suture and needle used for UCSs. We only included studies describing the sutures in the systematic review.

Results: We found 196 studies and included 76 (38.8\%) in our analysis. We collected data on maternal outcomes for 924 patients and categorized them. Of the 76 studies, suture sizes 0, 1, and 2 were used in 6, 44, and 32 articles, respectively (some studies used multiple sutures). Of the 45 studies mentioning the needles, curved and straight needles were used in 35 and 10, respectively. The results of our review revealed that about $80 \%$ of previous articles used Catgut and Polyglactin 910 sutures. Because no studies that compared the efficacy of different size of sutures were identified, we investigated the differences using the cases reported in previous studies mentioned above. In the first analysis, we compared the uterine preservation rate between size 1 and size 2 sutures. We found no significant difference in uterine preservation rate (92.8\%: size 1 vs. 94.2\%: size $2, p>0.05$ ) but found significant difference in transfusion rate $(62.4 \%$ vs. $79.1 \%, p<0.01)$. With the hypothesis that non-transfusion cases were less severe, we excluded these cases from second analysis. Although our second analysis of only Catgut or Polyglactin showed strong selection bias, we observed that uterine preservation rate was significantly higher in cases with size 2 suture than in those with size 1 suture $(86.9 \%$ vs. $93.5 \%, p=0.033)$.

Conclusions: Our systematic review showed that approximately $80 \%$ of cases were treated by Catgut and Polyglactin 910. Due to the heterogeneity of cases included in this review, it is difficult to estimate which suture is better for UCSS. More robust studies are necessary to enable the identification of the superior suture for performing UCSS.
\end{abstract}

Keywords: Uterine compression suture, Needle, Suture size

\footnotetext{
* Correspondence: zacky@gyne.med.osaka-u.ac.jp;

ahayapyon1020@yahoo.co.jp

1 Department of Obstetrics and Gynecology, Osaka University Graduate

School of Medicine, 2-2 Yamadaoka, Suita, Osaka 565-0871, Japan

Full list of author information is available at the end of the article
}

C The Author(s). 2019 Open Access This article is distributed under the terms of the Creative Commons Attribution 4.0 International License (http://creativecommons.org/licenses/by/4.0/), which permits unrestricted use, distribution, and reproduction in any medium, provided you give appropriate credit to the original author(s) and the source, provide a link to the Creative Commons license, and indicate if changes were made. The Creative Commons Public Domain Dedication waiver (http://creativecommons.org/publicdomain/zero/1.0/) applies to the data made available in this article, unless otherwise stated. 


\section{Background}

Severe hemorrhage after birth is known as postpartum hemorrhage $(\mathrm{PPH})$, and it is a major reason for maternal death worldwide, with an incidence in 1-2\% of live births. Uterine compression sutures (UCSs) have been indicated to manage PPH [1-3]. Reported UCS techniques have shown similar success rates (76-100\%) for uterine preservation [4-14], and these reports have proven that UCSs are essential treatment options for $\mathrm{PPH}$.

Although many UCS techniques are considered valuable, differences in the efficacies of different needles and sutures have not been well studied as most studies have focused on modification of the UCS procedure and in turn disregarded the influence of the choice of needles and sutures used. Thus, we focused on the efficacies of needles and sutures used for UCSs to identify the best ones for UCSs and help improve patient outcomes. In our previous study, we performed literature search on suture and needle types used for UCSs; however, we only investigated the results on dedicated needles for UCSs [15].

Here, we present the results of our systematic literature review to compare the efficacy of different types of needle and suture used for UCSs.

\section{Methods}

\section{Factors}

We conducted a systematic search of articles published between January 1, 1997, and May 31, 2017, using PubMed and Scopus databases, as performed in our previous study $[15,16]$. We included only English articles and excluded articles reported before 1997. The search strategy involved the use of these keywords: uterine compression suture, B-Lynch, and uterine preservation surgery. Articles on B-Lynch sutures were also included.

\section{Article retrieval}

We reviewed the retrieved articles following the Preferred Reporting Items for Systematic Reviews and MetaAnalyses guidelines [17]. Two authors (SM and MJ) independently performed the study selection (screening of titles, abstracts, and full texts of relevant articles) as described [18]. Many articles we found had insufficient information regarding the needles; thus, we chose all those that included information on sutures with or without needles for UCSs (suture sizes were those defined by the United States Pharmacopeia). We excluded articles that did not mention the suture type or uterine preservation rate. The outcomes and complication rates for each suture type were not available in all articles.

Given the reported similarity in high uterine preservation rates among the types of UCS [4], we assumed equivalent efficacies among UCS techniques in our analysis.

As mentioned in our previous study, our institution standardized the timing for performing UCSs to avoid performing unnecessary sutures [6]. However, most publications have failed to mention UCS timings, and these certainly vary among facilities; thus, comparing the different needle and suture types to determine the efficacy of UCSs was challenging. We limited our analysis to transfused cases and to those using Polyglactin 910 or Catgut sutures for maximum alignment, to compare the uterine preservation rates between suture sizes 1 and 2 in studies with similar conditions. Thus, we assumed that all patients who underwent hysterectomy had received transfusions.

Our primary aim with this study was to investigate the efficacies of UCSs with different needle and suture types. Our secondary aim was to reveal the needle and suture types used in previous studies.

\section{Statistical analysis}

Statistical analyses were performed using JMP Pro (version 13.1; SAS Institute, Tokyo, Japan). Categorical variables were analyzed using $X^{2}$ and Fisher's tests and continuous variables using one-way analysis of variance. $P$-value $<0.05$ was considered to indicate statistical significance.

\section{Results}

Search results

Figure 1 presents a flow diagram of the literature search. Of the 196 nonduplicate records identified from the electronic database search and other sources, we retrieved 196 full-text articles after screening the titles and abstracts. After excluding the articles that did not meet the inclusion criteria (56 case series and 20 case reports), we included 76 articles for analysis. Additional file 1: Table S1 and Additional file 2: Table S2 provide detailed information and references of all included articles.

\section{Results of the literature review on the type of needles and sutures used for UCS}

All 76 articles described UCSs with suture size and 45 included needle types. Of the 76 studies, suture sizes 0 , 1 , and 2 were used in 6, 44, and 32 articles, respectively (some studies included procedures with different suture and needle types; Table 1 ). Of the 45 studies that included needle types, 35 used curved needles and 10 straight needles. Sutures size 1 and curved needles were used most often. Determining the association between the procedure efficacy and the needle types was difficult because various types of needles were used during the UCSs; thus, we focused on the differences among suture types. The results of our review showed that about $80 \%$ of the studies included used Catgut or Polyglactin 910 sutures (19 and 45 studies, respectively). 


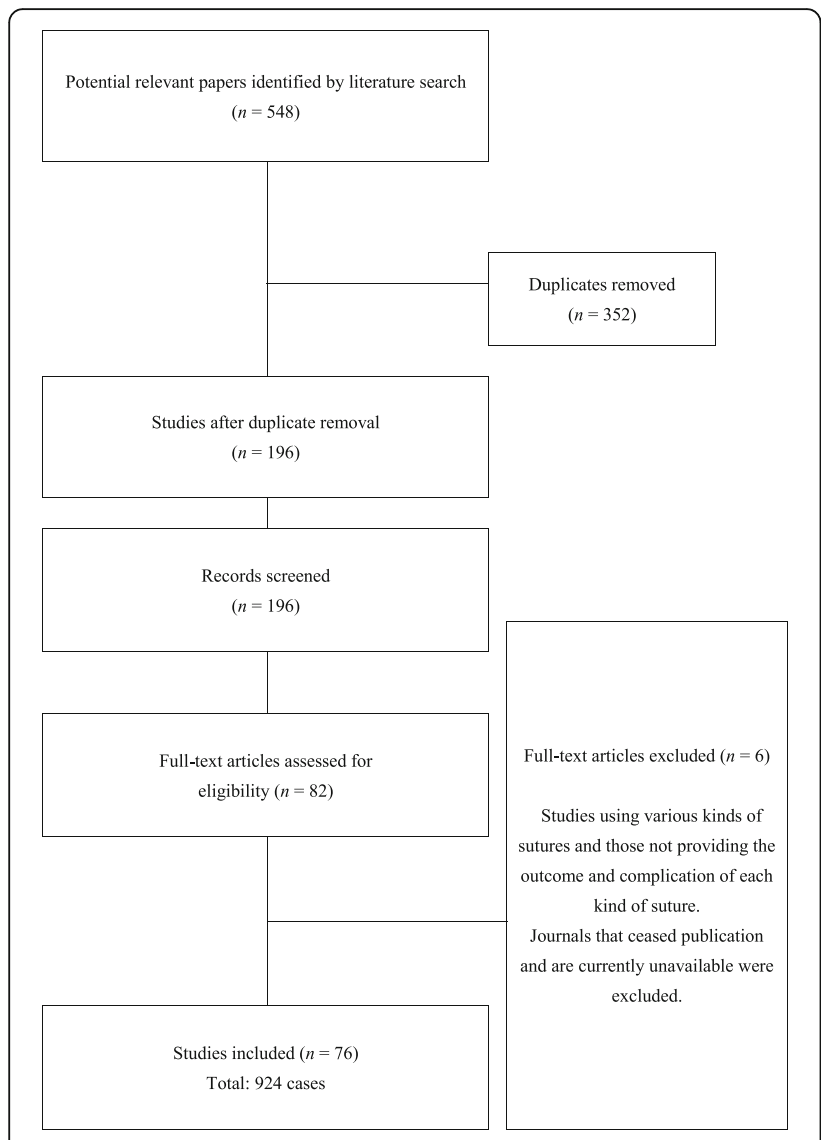

Fig. 1 Flow diagram of study selection for the systematic review

\section{Transfusion rate}

The transfusion rates ranged from 0 to $100 \%$ and varied between studies, with 42 studies mentioning about transfusion rate (including $\geq 5$ cases of UCS). Of the 42 studies, $26(61.9 \%)$ showed more than $80 \%$ transfusion rate, 13 (31.0\%) showed $10-79 \%$, and $3(7.1 \%)$ showed $0-9 \%$.

\section{Comparison of the UCS surgical outcomes for different suture sizes}

Table 2 shows the surgical outcomes for each suture size. A significantly lower transfusion rate was observed in the group of UCSs performed with size 1 sutures than in the group of UCSs performed with size 2 sutures $(62.4 \%$ vs. $79.1 \%$, respectively, $p<0.01)$. However, the uterine preservation rates did not significantly differ between the groups $(92.8 \%$ vs. $94.2 \%, p=0.249)$. Four uterine necroses $(0.67 \%$ of the total 596 UCSs) occurred in procedures performed with size 1 sutures, and none occurred in those performed with size 2 sutures. As shown in Table 2, we found no significant differences in the rates of severe complications (complications needing surgical interventions) after procedures performed with either size 1 or size 2 sutures $(p=0.332)$.
We performed second analysis by limiting our analyses to cases requiring transfusions with the hypothesis that non-transfusion cases were less severe. We only included the cases using Polyglactin 910 or Catgut sutures in this analysis. The uterine preservation rate was significantly higher in the procedures performed with size 2 sutures than in those performed with size 1 sutures (Table 3; $86.9 \%$ vs. $93.5 \%$, respectively, $p=0.033$ ). Next, we compared the outcomes after size 1 and 2 suture UCSs (Polyglactin 910 and catgut) in cases of uterine atony. There was no significant difference in the rates of transfusion and uterine preservation in the analysis of limited transfusion cases of uterine atony $(89.3 \%$ vs. $92.5 \%, p=$ 0.428) (Table 3 and Additional file 3: Table S3).

\section{Discussion}

The key finding of our study was that approximately $80 \%$ cases were treated by Catgut and Polyglactin 910 sutures with size 1 or 2 for UCSs. This systematic review is the first to compare different sutures (sizes 1 or 2) used for UCSs. However, we conclude that it is difficult to estimate which suture is better for UCSs due to the heterogeneity of cases included in this review.

Our review also found that transfusion rates varied from 0 to $100 \%$ and more than half of the studies showed high transfusion rate $(\geq 80 \%)$. First, UCS was indicated only in severe hemorrhage cases [5]. However, recently, UCS is used in mild cases, even though "prophylactic UCS" is currently performed [19]. We considered that these differences might reflect the differences in the initiation times of UCSs in each study.

We compared different types of sutures used for UCSs; however, the analysis was difficult because when performing UCSs for mild cases, the transfusion rate was low and the uterine preservation rate may have been high with the use of UCSs. For example, El Refaeey AEA et al investigated 108 cases [20], which included the largest number of UCSs in this review, and reported the rates of transfusion and uterine preservation of only $9.3 \%$ (10 of 108) and $100 \%(108 / 108)$ cases, respectively. The authors performed "early" intervention, resulting in the treatment of less severe cases and reduction of the rate of transfusion.

According to our results, the transfusion rate in UCS cases performed with size 1 sutures was significantly lower than that in cases performed with size 2 sutures $(62.4 \%$ vs. $79.1 \%$, respectively, $p<0.01$ ), but the uterine preservation rates were similar between the same groups $(92.8 \%$ vs. $94.2 \%$, respectively, $p=0.249$ ). Therefore, we attempted to limit the analysis of uterine preservation rates to transfused cases and to only analyze cases using Polyglactin 910 or Catgut sutures to reduce the difference in severity between the cases using size 1 and 2 sutures. Although this analysis showed a strong bias, sub-analysis showed that the uterine preservation rate in transfused cases was 
Table 1 Summary of published results on the review of the type of sutures used for uterine compression sutures

\begin{tabular}{|c|c|c|c|c|c|c|c|}
\hline \multirow{2}{*}{$\begin{array}{l}\text { Suture } \\
\text { size }\end{array}$} & \multirow[t]{2}{*}{ Suture material } & \multirow{2}{*}{$\begin{array}{l}\text { Number or } \\
\text { studiesa }\end{array}$} & \multirow{2}{*}{$\begin{array}{l}\text { Total cases included } \\
\text { in the studies }\end{array}$} & & \multicolumn{3}{|c|}{ Needle types } \\
\hline & & & & & Curved & Straight & NA \\
\hline \multirow[t]{5}{*}{ No. 0} & Polyglactin 910 & 4 & 13 & Shape & 3 & 0 & 0 \\
\hline & & & & Bodied & 0 & 0 & 0 \\
\hline & & & & NA & 0 & 0 & 1 \\
\hline & Catgut & 1 & 5 & & NA & & \\
\hline & Poliglecaprone 25 & 1 & 1 & NA & 0 & 1 & 0 \\
\hline \multirow[t]{12}{*}{ No. 1} & Polyglactin 910 & 25 & 355 & Shape & 3 & 0 & 0 \\
\hline & & & & Bodied & 5 & 1 & 0 \\
\hline & & & & NA & 6 & 1 & 9 \\
\hline & Catgut & 7 & 85 & Shape & 1 & 1 & 0 \\
\hline & & & & Bodied & 2 & 0 & 0 \\
\hline & & & & NA & 0 & 1 & 2 \\
\hline & Polydioxanone & 2 & 2 & & NA & & \\
\hline & Poliglecaprone 25 & 3 & 36 & Bodied & 1 & 0 & 0 \\
\hline & & & & NA & 0 & 0 & 2 \\
\hline & Others & 7 & 98 & Shape & 2 & 1 & 0 \\
\hline & & & & Bodied & 1 & 0 & 0 \\
\hline & & & & NA & 0 & 0 & 3 \\
\hline \multirow[t]{10}{*}{ No. 2} & Polyglactin 910 & 16 & 128 & Shape & 0 & 0 & 0 \\
\hline & & & & Bodied & 2 & 0 & 0 \\
\hline & & & & NA & 3 & 2 & 9 \\
\hline & Catgut & 11 & 155 & Shape & 0 & 0 & 0 \\
\hline & & & & Bodied & 3 & 0 & 0 \\
\hline & & & & NA & 2 & 1 & 5 \\
\hline & Polydioxanone & 1 & 1 & & NA & & \\
\hline & Others & 4 & 25 & Shape & 0 & 0 & 0 \\
\hline & & & & Bodied & 1 & 0 & 0 \\
\hline & & & & NA & 0 & 1 & 2 \\
\hline
\end{tabular}

${ }^{\mathrm{a} S o m e}$ studies overlapped

higher when performed with size 2 sutures than with size 1 sutures (86.9\%: size 1 vs. 93.5\%: size 2, $p=0.033$ ). Moreover, there were no between-group differences in terms of complication rate.

Although our systematic review failed to investigate differences in terms of efficacy among needle types, we found that three dedicated needles have already been reported: poliglecaprone 25, No.1 suture made by Ethicon EndoSurgery (W3709) for B-Lynch technique [21, 22]; a curved needle with customized sizable, reported by Yano et al. for MY suture [23]; and a straight super blunt needle reported by Matsuzaki et al. [15] These studies failed to fulfill our criteria and were excluded. Whether dedicated needles can improve the uterine preservation rate remains unclear.

The strength of our study lies in it being the first review to compare the efficacies of different needles and sutures for UCSs. Although it was difficult to conclude the superiority of needle and suture for UCS, our data may be useful to investigate the efficacy of needles and sutures in future studies. Second, our results also provide useful information, such as the needle and suture types being used for UCS as well as the transfusion rates of previous studies.

We are aware of our study's limitations. First, because we did not investigate the efficacy of different UCSs, the efficacy of different sutures was analyzed under the assumption that UCS techniques are equally effective and show the same uterine preservation rate. This assumption may have strongly biased the result of our analysis. Second, we could not investigate the efficacies of different sutures under the same conditions because PPH often occurs because of different causes and because UCS timing varies according to clinicians' judgment. To adjust the severity of conditions, 
Table 2 Review of the suture types used for uterine compression sutures in previous studies (all cases)

\begin{tabular}{|c|c|c|c|c|c|c|}
\hline Suture size & Suture material & $\begin{array}{l}\text { Total cases included } \\
\text { in the studies }\end{array}$ & $\begin{array}{l}\text { Estimated mean } \\
\text { blood loss (ml) }\end{array}$ & Transfusion rate & Uterine preservation rate & Severe complication rate \\
\hline \multirow[t]{2}{*}{ No. 0} & Polyglactin 910 & 13 & 3082 & 9/10 (90\%) & 13/13 (100\%) & 0 \\
\hline & Catgut & 5 & 2830 & $5 / 5(100 \%)$ & $5 / 5(100 \%)$ & 0 \\
\hline \multirow[t]{4}{*}{ No. 1} & Polyglactin 910 & 375 & 1921 & $211 / 372(56.7 \%)$ & $342 / 375(91.2 \%)$ & $3 / 375(0.8 \%)$ \\
\hline & Catgut & 85 & 1380 & $34 / 40$ (85.0\%) & $82 / 83(98.8 \%)^{b}$ & $1 / 83(1.2 \%)^{b}$ \\
\hline & Others & 136 & 2309 & 97/136 (71.3\%) & 127/136 (93.4\%) & 1/136(0.74\%) \\
\hline & Total & 596 & 2039 & $342 / 548(62.4 \%)$ & $551 / 594(92.8)^{b}$ & $5 / 594(0.91 \%)^{b}$ \\
\hline \multirow[t]{4}{*}{ No. 2} & Polyglactin 910 & 128 & 2135 & 92/102 (90.2\%) & 120/128 (93.8\%) & 0/128 (0\%) \\
\hline & Catgut & 155 & 2361 & 78/118 (66.1\%) & 146/155 (94.2\%) & 0/124 (0\%) \\
\hline & Others & 26 & 2531 & 19/19 (100\%) & 25/26 (96.2\%) & 0/19 (0\%) \\
\hline & Total & 309 & 2249 & 189/239 (79.1\%) & 291/309 (94.2\%) & $0 / 271(0 \%)$ \\
\hline
\end{tabular}

${ }^{a}$ Severe complications are defined as the need for surgical intervention to treat complications due to uterine compression sutures

${ }^{\mathrm{b}}$ Two maternal deaths due to the hypertention disorder of pregnancy were excluded

we limited the analysis of uterine preservation rate to cases requiring transfusions; however, this selection may also have caused biases.

Third, we could not classify the PPH cases according to their causes, and we had to individually determine the efficacies of sutures. Moreover, the relationship between uterine preservation rate and different needles or suture was not compared in the same study, negating the feasibility of a needle- or suture-specific discussion. Fourth, we could not investigate or exclude the effect of adjunctive treatments such as uterine artery ligation for UCS; thus, unmeasured bias may exist in the analysis. Fifth, because previous studies lacked detailed information, we could not determine the timing of transfusion. Therefore, these results may be biased because our hypothesis was that nontransfusion cases exhibit less severity.

As mentioned above, our study has strong biases; however, we believe it is an acceptable method. In order to resolve these biases, better study designs, such as casecontrol and cohort studies, and perhaps even more robust studies, such as randomized control trials, are expected to provide better evidence regarding the needles and sutures used for UCSs.

\section{Conclusions}

A systematic review determining the associations between size 1 and size 2 sutures used for UCS and its outcomes in postpartum hemorrhage showed that approximately $80 \%$

Table 3 Comparison of uterine preservation rate among different suture sizes

\begin{tabular}{|c|c|c|c|c|c|c|}
\hline Suture size & Suture material & $\begin{array}{l}\text { Total cases included } \\
\text { in the studies }\end{array}$ & Mean blood loss (ml) & Transfusion rate & Uterine preservation & Severe complication rate ${ }^{a}$ \\
\hline \multirow[t]{3}{*}{ No. 1} & Total & $594^{b}$ & 2039 & $342 / 548(62.4 \%)^{c}$ & $551 / 594(92.8 \%)^{d}$ & $5 / 594(0.84 \%)^{e}$ \\
\hline & \multicolumn{2}{|c|}{$\begin{array}{l}\text { Limited transfusion cases } \\
\text { (Polyglactin } 910 \text { and catgut) }\end{array}$} & & & $213 / 245(86.9 \%)^{f}$ & \\
\hline & \multicolumn{2}{|c|}{$\begin{array}{l}\text { Limited transfusion and } \\
\text { uterine atony cases } \\
\text { (Polyglactin } 910 \text { and catgut) }\end{array}$} & & & $142 / 159(89.3 \%)^{9}$ & \\
\hline \multirow[t]{3}{*}{ No. 2} & Total & 309 & 2249 & $189 / 239(79.1 \%)^{c}$ & $291 / 309(94.2 \%)^{d}$ & $0 / 309(0 \%)^{e}$ \\
\hline & \multicolumn{2}{|c|}{$\begin{array}{l}\text { Limited transfusion cases } \\
\text { (Polyglactin } 910 \text { and catgut) }\end{array}$} & & & $159 / 170(93.5 \%)^{f}$ & \\
\hline & \multicolumn{2}{|c|}{$\begin{array}{l}\text { Limited transfusion and uterine } \\
\text { atony cases (Polyglactin } 910 \\
\text { and catqut) }\end{array}$} & & & $135 / 146(92.5 \%)^{9}$ & \\
\hline
\end{tabular}

Bold indicates statistical significance

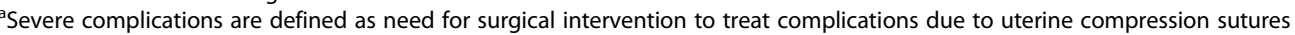

${ }^{\mathrm{b}}$ Two maternal deaths due to the hypertention disorder of pregnancy were excluded

Statistical analysis was performed among $b, c, d$, and e. The $p$ values are listed below

${ }^{c} p=0.000$

$\mathrm{d}_{p}=0.249$

${ }^{\mathrm{e}} p=0.332$

$f^{p} p=0.033$

${ }^{\mathrm{g}} p=0.428$ 
of cases were treated by Catgut and Polyglactin 910. We also revealed that it is difficult to investigate the efficacy of UCSs among the different needles and sutures used based on the current knowledge. Comparisons between size 1 and size 2 sutures in more robust studies are necessary to enable the identification of the superior suture for performing UCSs.

\section{Supplementary information}

Supplementary information accompanies this paper at https://doi.org/10. 1186/s12893-019-0660-Z

Additional file 1: Table S1. Detailed information of the sutures and needles in published studies

Additional file 2: Table S2. References of all publications on uterine compression sutures that satisfied our criteria

Additional file 3: Table S3 Comparison of uterine preservation rate among different suture sizes in uterine atony cases

\section{Abbreviations}

PPH: Postpartum hemorrhage; UCS: Uterine compression suture

\section{Acknowledgements}

The authors would like to thank Kanako Sakiyama and Hazuki Abe for administrative assistance in the preparation of our review.

\section{Authors' contributions}

ShM and AK is the corresponding author. SM, JM, AK, TM, EK, TTa and TK designed the study. ShM, SaM, JM, TH and SN collected the data. ShM, MJ, SaM, TTa, and TTo analyzed the data. ShM, JM, TH, SN and TK made interpretation of data. ShM, JM and AK wrote the manuscript. ShM, JM, AK and TK reviewed the manuscript. All authors read and approved the final manuscript.

\section{Funding}

The English editing fee was supported by a Grant-in-Aid for Scientific Research (C) (17 K11278 to Shinya Matsuzaki). No financial support was received in the design of the study and collection, analysis, and interpretation of data and writing the manuscript.

\section{Availability of data and materials}

The dataset used and/or analyzed during the current study are available from the corresponding author on reasonable request.

\section{Ethics approval and consent to participate}

Not applicable.

\section{Consent for publication}

Not applicable.

\section{Competing interests}

Shinya Matsuzaki is an Associate Editor for BMC Pregnancy and Childbirth The authors declare no conflicts of interest (COI) about this study. All of authors have no competing financial interests regarding this study.

\section{Author details}

'Department of Obstetrics and Gynecology, Osaka University Graduate School of Medicine, 2-2 Yamadaoka, Suita, Osaka 565-0871, Japan.

${ }^{2}$ Department of Obstetrics and Gynecology, Otemae Hospital, Osaka, Japan.

Received: 18 March 2019 Accepted: 29 November 2019

Published online: 16 December 2019

\section{References}

1. Maswime S, Buchmann E. A systematic review of maternal near miss and mortality due to postpartum hemorrhage. Int J Gynaecol Obstet. 2017;137: $1-7$.
2. Committee on Practice B-O. Practice Bulletin No. 183: Postpartum Hemorrhage. Obstet Gynecol. 2017;130:e168-e86.

3. Matsuzaki S, Endo M, Ueda Y, et al. A case of acute Sheehan's syndrome and literature review: a rare but life-threatening complication of postpartum hemorrhage. BMC Pregnancy Childbirth. 2017;17:188.

4. Matsubara S, Yano H, Ohkuchi A, Kuwata T, Usui R, Suzuki M. Uterine compression sutures for postpartum hemorrhage: an overview. Acta Obstet Gynecol Scand. 2013;92:378-85.

5. B-Lynch C, Coker A, Lawal AH, Abu J, Cowen MJ. The B-Lynch surgical technique for the control of massive postpartum haemorrhage: an alternative to hysterectomy? Five cases reported. Br J Obstet Gynaecol. 1997;104:372-5

6. Suzuki Y, Matsuzaki S, Mimura K, et al. Investigation of perioperative complications associated with use of uterine compression sutures. Int J Gynaecol Obstet. 2017;139:28-33.

7. Nishikawa A, Matsuzaki S, Mimura K, Kanagawa T, Kimura T. Short interpregnancy interval after B-Lynch uterine compression suture: a case report. Clin Exp Obstet Gynecol. 2016;43:434-6.

8. Gonzalez JM, Ofori E, Burd I, Chai J, Scholler N, Elovitz MA. Maternal mortality from systemic illness: unraveling the contribution of the immune response. Am J Obstet Gynecol. 2009;200:430 e1-8.

9. Hayman RG, Arulkumaran S, Steer PJ. Uterine compression sutures: surgical management of postpartum hemorrhage. Obstet Gynecol. 2002;99:502-6.

10. Cho JH, Jun HS, Lee CN. Hemostatic suturing technique for uterine bleeding during cesarean delivery. Obstet Gynecol. 2000;96:129-31.

11. Matsubara S, Kuwata T, Baba Y, et al. A novel 'uterine sandwich' for haemorrhage at caesarean section for placenta praevia. Aust N Z J Obstet Gynaecol. 2014;54:283-6.

12. Mohamed MA, Mohammed AH. Parallel vertical compression sutures to control bleeding in cases of placenta previa and accreta. J Matern Fetal Neonatal Med. 2019:32:641-5.

13. Takahashi H, Baba Y, Usui R, et al. Matsubara-Yano suture: a simple uterine compression suture for postpartum hemorrhage during cesarean section. Arch Gynecol Obstet. 2019;299:113-21.

14. Matsuzaki S, Tanaka M, Kimura T. Response to "Letter to "cervical varix complicated by placenta previa: a case report and literature review": a novel simple abdominal traction stitch (Matsubara) to hold the Bakri balloon'. J Obstet Gynaecol Res. 2016;42:1627-8.

15. Matsuzaki S, Endo M, Tomimatsu T, et al. New dedicated blunt straight needles and sutures for uterine compression sutures: a retrospective study and literature review. BMC Surg. 2019;19:33.

16. Matsuzaki S, Matsuzaki S, Ueda Y, et al. A case report and literature review of Midtrimester termination of pregnancy complicated by placenta Previa and placenta Accreta. AJP Rep. 2015:5:e6-e11.

17. Moher D, Liberati A, Tetzlaff J, Altman DG, Group P. Preferred reporting items for systematic reviews and meta-analyses: the PRISMA statement. BMJ. 2009;339:b2535.

18. Matsuzaki S, Yoshino K, Endo M, Kakigano A, Takiuchi T, Kimura T. Conservative management of placenta percreta. Int J Gynaecol Obstet. 2018;140:299-306.

19. Vijayasree M. Efficacy of prophylactic B-Lynch suture during lower segment caesarian section in high risk patients for atonic postpartum Haemorrhage. Kathmandu Univ Med J (KUMJ). 2016;14:9-12.

20. El-Refaeey AA, Gibreel A, Fawzy M. Novel modification of B-Lynch uterine compression sutures for management of atonic postpartum hemorrhage: W uterine compression sutures. J Obstet Gynaecol Res. 2014;40:387-91.

21. Price $\mathrm{N}$, Lynch $\mathrm{C}$. Uterine necrosis following B-Lynch suture for primary postpartum haemorrhage. BJOG. 2006;113:1341 author reply 42.

22. Price N, B-Lynch C. Technical description of the B-Lynch brace suture for treatment of massive postpartum hemorrhage and review of published cases. Int J Fertil Womens Med. 2005:50:148-63.

23. Yano H, Kuwata T, Kosuge S, Matsubara S. A long curved needle with a large radius for uterine compression suture. Acta Obstet Gynecol Scand. 2013;92:988-9.

\section{Publisher's Note}

Springer Nature remains neutral with regard to jurisdictional claims in published maps and institutional affiliations. 